It can also readily be shown that one may successively find the $a_{n}^{\prime}$ (and hence $a_{n}$ ) so that

$$
\psi_{y}(x, 0)=-1 \quad \text { on } \quad 0<x<A .
$$

The series will fail to converge, of course, at $x=A$. However, $a_{0}$ can readily be shown to exceed 1.11 (the value obtained for the semi-infinite plate) and thus the finite plate consideration does not make the unmodified Oseen procedure more valid. In fact, one might use the modified procedure to improve the finite plate solution although, for this problem, we do not know the velocity gradient at the plate as we did for the long plate. In any event, the numerical evaluation of the velocity field will not be pursued here in any detail.

\title{
AN ITERATIVE METHOD FOR FINDING THE SMALLEST EIGENVALUE OF A MATRIX*
}

\author{
By JOHN B. LOHMAN (Brown University)
}

The eigenvalues and eigenvectors of a matrix $A$ are often found by repeated premultiplication of an arbitrary column vector $v$ by the matrix $A$. The products $A^{n} v$ converge to the eigenvector $v_{1}$ corresponding to the eigenvalue $\lambda_{1}$ largest in absolute magnitude; convergence is rapid if $\lambda_{1}$ is much larger than any other eigenvalue of $A$.

If one already has approximate values of the eigenvalues of $A$, this iterative method can be modified to give more rapid convergence. Aitken ${ }^{1}$ suggests subtracting a scalar matrix $a I$ ( $I$ denotes the unit matrix) from $A ; a$ is chosen so as to give the maximum separation of $\lambda_{1}$. Kincaid ${ }^{2}$ carries out the iteration using a polynomial $P(A)$ in $A$, in place of $A$ itself; $P(A)$ is so chosen that the eigenvalues not immediately sought are as nearly zero as possible.

Iteration for the smallest eigenvalue of a matrix $A$ is commonly performed using the inverse matrix $A^{-1}$. This note deseribes a modified procedure leading to more rapid convergence.

Let the eigenvalues of $A$ be $.22, .4,1,2$, and assume we know them only to within $10 \%$. Simple inversion of $A$ yields $A^{-1}$ with eigenvalues $4.5,2.5,1, .5$; the largest eigenvalue of $A^{-1}$ (corresponding to the smallest eigenvalue of $A$ ) is 1.8 times as large as the second-largest. Assume we believe the smallest eigenvalue of $A$ to be .2 instead of .22; subtract the scalar matrix .2I from $A$, and then invert; the resulting matrix $(A-.2 \mathrm{I})^{-1}$ has eigenvalues $50,5,1.25, .56$. But we can still improve the separation by subtracting another scalar matrix. We choose the mean of the second largest and the smallest eigenvalues (a different choice would be appropriate if some eigenvalues were negative), for maximum separation of the largest and the second largest eigenvalues. Our final modified matrix, $(A-.2 \mathrm{I})^{-1}-2.78 \mathrm{I}$, has eigenvalues $47.22,2.22,-1.53,-2.22$. The ratio of largest to second largest eigenvalue has been increased from 1.8 to 21.3.

Since the modified matrix has all but one of its eigenvalues tightly clustered about zero, it is not a suitable form from which to determine these other eigenvalues.

${ }^{*}$ Received July 22, 1948. This work was supported under Navy contract.

${ }^{1}$ A. C. Aitken, Proc. Roy. Soc. Edinburgh 57, 269-270 (1937).

${ }^{2}$ W. M. Kincaid, Q. Appl. Math. 5, 320-345 (1947). 